\title{
Male breast cancer - a single center experience
}

\author{
BRANISLAV BYSTRICKY, FILIP KOHUTEK and ANDREJ ROSIK \\ Oncology Department, Faculty Hospital Trenčín, 91171 Trenčín, Slovak Republic
}

Received January 29, 2016; Accepted June 10, 2016

DOI: $10.3892 / \mathrm{ol} .2016 .4807$

\begin{abstract}
Due to its rarity, male breast cancer remains a poorly characterized disease. The present study obtained retrospective clinicopathological data, treatment patterns and outcomes for all male patients diagnosed with breast cancer in the Oncology Department, Faculty Hospital Trenčín (Trenčín, Slovakia) over the last 21 years from January 1995 to December 2015. A total of 21 patients with male breast cancer were analyzed, with a median patient age of 65.6 years. Two patients were diagnosed with lobular invasive cancer; all others were diagnosed with cancer of a ductal origin. One patient presented with metastatic disease in the pleural cavity. The primary tumors in 8 patients were staged as pT1, whilst 6 patients were staged as pT2 and 7 as pT4. Axillary lymph node involvement was present in 11 patients (52\%) and 15 patients were hormone receptor-positive $(83 \%)$. All but 1 patient underwent mastectomy and surgical staging of the axilla. Adjuvant chemotherapy, radiotherapy and hormone treatment was administered in the same manner as breast cancer treatment in female patients. The median follow-up time was 4.5 years. The 5- and 10 -year overall survival rates were 87 and $74 \%$, respectively, and the estimated median disease-free survival for the same population was 9.5 years (95\% confidence interval, 6.2-14.6). The survival rates reported in the present retrospective study are comparable with previously published studies. In addition, the current study reported predominant hormone-positive characteristics and rare expression of human epidermal growth factor receptor 2. However, further multi-institutional trials are required to allow for informed treatment decisions in this uncommon disease.
\end{abstract}

Correspondence to: Dr Branislav Bystricky, Oncology Department, Faculty Hospital Trenčín, Legionarska 28, 91171 Trenčín, Slovak Republic

E-mail: brano.bystricky@gmail.com

Abbreviations: OS, overall survival; DFS, disease-free survival; ER, estrogen receptor; PR, progesterone receptor; HER2, human epidermal growth factor receptor 2; E2, estradiol; LHRH, luteinizing hormone-releasing hormone agonist; FSH, follicle-stimulating hormone

Key words: male breast cancer, chemotherapy, hormonal treatment, retrospective study

\section{Introduction}

Male breast cancer is a rare disease accounting for $<1 \%$ of all diagnosed breast cases (1). The only ethnic group with a higher than average incidence of male breast cancer is Jewish men, regardless of their residence (2). A previous study determined a strong family predisposition for the disease, with an odds ratio of 3.3 (3). It is recognized that breast cancer (BRCA) mutations may be detected in a significant portion of male breast cancer cases (primarily BRCA2) and the National Comprehensive Cancer Network guidelines recommend genetic testing for such patients (4). It has been suggested that male breast cancer is associated with a poorer prognosis compared with females, largely due to delays in diagnosis, lack of clinical care pathways and associated comorbidities due to the advanced age of patients (5). A recent comparative analysis of menopausal status in female patients with breast cancer concluded poorer outcomes for male patients with breast cancer compared with postmenopausal females and similar outcomes to premenopausal female patients with breast cancer (6). Recommendations for treatment in males are extrapolated from extensive evidence in women. Due to lack of breast tissue, simple mastectomy is commonly the preferred surgical procedure. Sentinel lymph node biopsy is feasible and is being performed more frequently in cases of male breast cancer. Given the absence of terminal lobules in the male breast, the disease is typically of a ductal type and hormone receptor-positive. Human epidermal growth factor receptor 2 (HER2)-positivity is rare, with a recent large multinational study reporting that this characteristic was observed in $<5 \%$ of cases (7). In the same study, a trend of improvement in overall survival (OS) was also observed over time in men with breast cancer (7).

The aim of the present study was to examine the clinical and pathological characteristics, treatment patterns and outcomes of a series of male patients with breast cancer, who were consecutively treated at a single institution from January 1995 to December 2015.

\section{Patients and methods}

Patients. The current study retrospectively reviewed medical records of all male patients diagnosed with breast cancer over the last 21 years from January 1995 to December 2015 at the Oncology Department, Faculty Hospital Trenčín (Trenčín, Slovakia), which serves a population of $>600,000$ inhabitants. All 21 cases identified in this time period were included in the 
present study. Individual patient records were reviewed and the relevant data was obtained, including stage at presentation [based on the 7th edition of the Tumor-Node-Metastasis (TNM) classification system] (8), histological classification and tumor biomarkers, and details regarding treatment and follow-up. The study was approved by the Ethics Committee of Faculty Hospital Trenčín.

Statistical analysis. Statistical analyses were performed using MedCalc 15.2 software (www.medcalc.org). The patients' characteristics were summarized using the median (range) for continuous variables and frequency (percentage) for categorical variables. Survival analysis was used only for patients treated with curative intent. Kaplan-Meier estimates were performed to calculate OS rate from the date of initial diagnosis to the date of last follow-up or mortality, and disease-free survival (DFS) was calculated from the date of initial diagnosis to the date of progression or mortality.

\section{Results}

Patient clinicopathological characteristics. All 21 male patients were diagnosed with invasive breast cancer during the study period, which was $<1 \%$ of the number of female cases diagnosed at the same time. The median age was 65.6 years (range, 51-86) and the majority of patients were over 60 years at diagnosis $(71 \%)$. As presented in Table I, the primary tumors in 8 patients were staged as pT1, whilst 6 patients were staged as pT2 and 7 as pT4. No patients presented with pT3 or Tis primary tumors. Axillary lymph node involvement was present in 11 patients $(52 \%)$ and 1 patient presented with metastatic disease (cytologically verified malignant pleural effusion) at the time of diagnosis. According to the TNM staging system (8), 6 patients were diagnosed with stage I disease, while 8 patients were stage II, 6 patients stage III and 1 patient stage IV. With regards to histological subtypes, invasive ductal cancer (invasive carcinomas of no special type) was present in 19 patients and invasive lobular cancer was present in 2 patients. Hormone receptor status was examined in 18 patients, of which $15(83 \%)$ were estrogen receptor (ER)-positive and 13 (72\%) were progesterone receptor (PR)-positive. Only 1 out of 11 patients examined for HER2 status was positive (9\%). Only 2 patients were triple (ER/PR/HER2)-negative. A total of 12 patients had grade 2 disease, while 5 patients had grade 1 and 4 had grade 3. BRCA testing was not performed on the majority of patients as reimbursement for male breast cancer was not in place until recent years. All 6 patients analyzed had normal karyotypes without any $B R C A$ mutations.

Treatment regimens. As presented in Table II, a total of 20 patients underwent primary surgical treatment (simple mastectomy) for localized breast cancer. The axilla was surgically staged in all 20 patients undergoing primary surgical treatment; axillary lymph node dissection was performed in 19 patients and sentinel node biopsy was performed in 1 patient. Adjuvant chemotherapy was administered to 9 patients. Indications for adjuvant systemic treatment were extrapolated from breast cancer in women. Anthracycline-based chemotherapy [adriamycin and cyclophosphamide, or 5-fluorouracil (5-FU), adriamycin and cyclophosphamide] was administered
Table I. Clinicopathological variables of male patients with invasive breast cancer.

\begin{tabular}{|c|c|}
\hline Variable & $\mathrm{n}(\%)$ \\
\hline \multicolumn{2}{|l|}{ Tumor size } \\
\hline pT1 & $8(38)$ \\
\hline pT2 & $6(29)$ \\
\hline pT3 & $0(0)$ \\
\hline pT4 & $7(33)$ \\
\hline \multicolumn{2}{|l|}{ Nodal status } \\
\hline No & $10(48)$ \\
\hline $\mathrm{N}+$ & $11(52)$ \\
\hline \multicolumn{2}{|l|}{ Metastases } \\
\hline Yes & $1(5)$ \\
\hline No & $20(95)$ \\
\hline \multicolumn{2}{|l|}{ Grade } \\
\hline 1 & $5(24)$ \\
\hline 2 & $12(57)$ \\
\hline 3 & $4(19)$ \\
\hline \multicolumn{2}{|l|}{ Histology } \\
\hline Ductal carcinoma in situ & $0(0)$ \\
\hline Invasive ductal carcinoma & $19(90)$ \\
\hline Invasive lobular carcinoma & $2(10)$ \\
\hline \multicolumn{2}{|l|}{ Estrogen receptor } \\
\hline Positive & $15(83)$ \\
\hline Negative & $3(17)$ \\
\hline N/A & $3(-)$ \\
\hline \multicolumn{2}{|l|}{ Progesterone receptor } \\
\hline Positive & $13(72)$ \\
\hline Negative & $5(28)$ \\
\hline $\mathrm{N} / \mathrm{A}$ & $3(-)$ \\
\hline \multicolumn{2}{|l|}{ HER2 } \\
\hline Positive & $1(9)$ \\
\hline Negative & $10(91)$ \\
\hline $\mathrm{N} / \mathrm{A}$ & $10(-)$ \\
\hline
\end{tabular}

Mean patient age was 65.6 years (range, 51-86 years). HER2, human epidermal growth factor receptor 2; N/A, not available.

to 5 patients, sequential chemotherapy based on anthracycline and taxane/trastuzumab was administered to 1 patient and cyclophosphamide, methotrexate and 5-FU was given to 3 patients (who had been diagnosed $>10$ years previously). Adjuvant radiotherapy was administered to 12 patients, with a total dose of 50 Gy (25 fractions over 5 weeks) with/without additional boosts to the tumor bed. Finally, 12 patients were treated with 5-year-long adjuvant hormonal treatment, consisting of 9 patients who were administered tamoxifen alone and 3 patients who underwent sequential hormonal therapy consisting of tamoxifen and aromatase inhibitor. One HER2-positive patient was administered adjuvant trastuzumab concurrently with adjuvant taxane treatment. Adjuvant trastuzumab was continued for 1 year. Progression to stage IV occurred in 3 patients, who were initially stage II 
Table II. Treatment details of male patients with invasive breast cancer.

\begin{tabular}{lc}
\hline Treatment & $\mathrm{n}(\%)$ \\
\hline Surgery (n=21) & \\
Mastectomy & $20(95)$ \\
Wide local excision & $0(0)$ \\
No breast surgery & $1(5)$ \\
Axillary lymph node dissection & $19(90)$ \\
Sentinel lymph node biopsy & $1(5)$ \\
No surgery in axilla & $1(5)$ \\
Adjuvant radiotherapy $(\mathrm{n}=20)$ & \\
Yes & $12(60)$ \\
No & $8(40)$ \\
Adjuvant chemotherapy (n=20) & \\
Yes & $9(45)$ \\
Anthracyclin-based & $5(56)$ \\
Antracyclin followed by taxane/trastuzumab & $1(11)$ \\
CMF & $3(33)$ \\
No & $3(55)$ \\
Adjuvant hormonal therapy (n=14) & \\
Yes & $12(86)$ \\
Tamoxifen & $9(75)$ \\
Aromatase inhibitors & $0(0)$ \\
Tamoxifen followed by aromatase inhibitors & $3(25)$ \\
No & $2(14)$ \\
\end{tabular}

For adjuvant treatment, patients excluding stage IV were considered. For hormonal treatment, only hormone receptor-positive patients were considered to be eligible. CMF, cyclophosphamide, methotrexate and 5-fluorouracil.

or III, following curative treatment. All of these patients were treated with palliative chemotherapy (anthracycline-based, taxane-based or capecitabine-based) and 2 also received additional hormonal therapy (tamoxifen). Palliative radiotherapy was delivered to 1 patient for skeletal metastases. One patient with initially metastatic disease to the pleura was treated with palliative chemotherapy (anthracycline-based) followed by tamoxifen, and upon progression was treated with taxane and aromatase inhibitors, in addition to luteinizing hormone-releasing hormone (LHRH) agonist.

Patient outcomes. A total of 4 patients succumbed during the median follow-up period of 4.5 years. Two patients succumbed due to progression of the disease and the cause of mortality regarding the other two patients is unknown. The 5- and 10 -year OS rates were 87 and $74 \%$, respectively (Fig. 1). The estimated median DFS for the same population was 9.5 years (95\% CI, 6.2-14.6) and 5-year DFS rate was 86\%.

\section{Discussion}

Male patients with breast cancer tend to present later in life than females and the incidence of male breast cancer, although

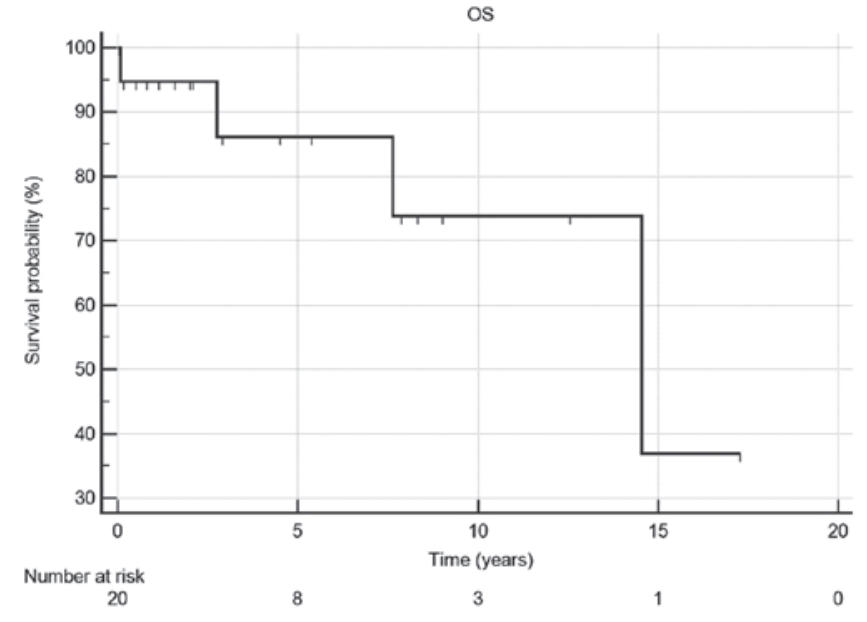

Figure 1. OS of all non-metastatic patients. OS, overall survival.

much lower than in females, is rising (1). Incidence of male breast cancer in Slovakia is $~ 0.8 \%$ of all female breast cancer cases, which is similar to that of Western Europe (9). Furthermore, $83 \%$ of the patients in the current study were hormone receptor-positive, while only $9 \%$ of patients were HER2-positive, which is in line with a recent retrospective study by Cardoso et al (7). Given this fact, the luminal A molecular subtype was predominantly observed in $58 \%$ of patients in the same study (7). Additional comparative biomarker studies of matched cohorts confirm a higher predominance of ER-positivity and luminal A subtypes in men (10). This knowledge is important for practicing oncologists, as all newly diagnosed males with breast cancer with negative hormonal status should undergo a thorough pathological review so that the opportunity for hormonal treatment is not missed. The present study observed only 2 cases of lobular carcinoma, which is a similar number reported by a previous population-based study (11). Surgical treatment of male breast cancer usually includes mastectomy, as cosmetic outcomes are typically less important than in women. Experience with sentinel lymph node biopsy in men is gaining wider acceptance among breast surgeons and is a recommended method of axillary staging of early cancer in women. The rarity of male breast cancer precludes prospective studies that may potentially guide the development of optimal treatment regimens. The decisions made regarding treatment in the present study were based on general guidelines for breast cancer in women, as recommended by the European Society for Medical Oncology (12). Limitations of the current study include the small number of patients diagnosed and treated over several years for a relatively rare disease. The majority of patients with positive axillary lymph nodes and/or pT4 primary tumor stage received adjuvant chemotherapy and chest-wall radiotherapy. All but 2 patients with ER-positive tumors received hormone treatment, initially with tamoxifen. One patient without hormonal treatment arrived from another institution and the other is currently finishing adjuvant chemotherapy. Tamoxifen remains the standard hormonal treatment in adjuvant and metastatic settings for male breast cancer (13). Hormonal treatment is preferred in palliative setting as outcomes are comparable with chemotherapy with less toxicity in women (14). The present 
study incorporated hormonal manipulation in the treatment strategies for all eligible palliative male breast cancer patients and there was no requirement for rapid tumor response.

Incorporation of aromatase inhibitors, a standard treatment approach in women, remains a contentious issue in male breast cancer. Aromatase, an estrogen synthetase, is a key enzyme in estrogen synthesis, which is expressed not only in the gonads and peripheral tissues (including fat and bone), but also intratumorally (15). In men, $60 \%$ of circulating estradiol (E2) is produced by the testes either directly or by conversion from testicular androgens (16). The remaining portion is derived from the conversion of adrenal androgens (16). Thus, the production of testicular and adrenal androgens, together with activity of aromatase enzyme, determine plasma estrogen concentration in men. LHRH agonists (also known as gonadotropin-releasing hormone agonists) are used in premenopausal women with breast cancer to reduce the production of ovarian estrogen and in castrate-sensitive prostate cancer in males. LHRH administration yields reduction of testosterone and estrogen produced by the testes (17). Combination with aromatase inhibitors may further block estrogen production via aromatization. Prospective and retrospective measurements of plasma E2, follicle-stimulating hormone (FSH) and testosterone in men treated with only aromatase inhibitors have been reported $(18,19)$, with results demonstrating that $\mathrm{E} 2$ levels decreased with treatment, but testosterone and FSH levels increased. These measurements confirmed a positive feedback loop between increasing FSH and testosterone. As testosterone is a substrate for aromatase, further E2 suppression may be achieved with LHRH analogs. A recent pooled analysis of 105 male breast cancer cases demonstrated the effectiveness of the aforementioned strategy (a 3-fold increase in clinical benefit rate) (20), and in a smaller analysis of 60 patients, a survival benefit was observed following use of the combination strategy (21). However, a phase II trial investigating this further was closed prematurely due to poor accrual (22). As this preclinical data suggests that complete hormonal suppression with aromatase inhibitors in men is not possible, they are (as monotherapy) not considered standard treatment in male breast cancer. All in all, tamoxifen continues to be administered as a standard adjuvant treatment in cases of male breast cancer, but dual treatment with LHRH agonists (or orchiectomy) and aromatase inhibitors is gaining ground in metastatic male breast cancer (23). Adjuvant chemotherapy is recommended for high-risk female patients with breast cancer, and by extrapolation, its effectiveness is assumed to apply to the treatment of males with the disease. In the present study, out of 14 patients with stage II or III disease, 5 patients did not receive adjuvant chemotherapy due to advanced age, patient or physician choice, and comorbidities. The 5- (87\%) and 10-year (74\%) OS rates and the 5-year DFS rate estimated in the current study are consistent with previously published studies $(13,24-26)$.

Due to the rarity of male breast cancer and largely anecdotal evidence from case reports, the International Male Breast Cancer Program was created under auspices of the European Organization for Research and Treatment of Cancer and the Translational Breast Cancer Research Consortium. In this program, a prospective cohort study analyzing a collection of DNA and tumor samples is planned. This program aims to advance the treatment of male breast cancer in a similar manner to how therapies have progressed for women with the disease. Male breast cancer is a rare disease and there are no prospective studies to inform current practice. As the disease has numerous similarities to breast cancer in women, treatment strategies are extrapolated from female breast cancer. Over the last 20 years, significant progress has been made in physician detection, investigation and treatment of male breast cancer. The encouraging results of the present study may give patients and professionals confidence in future treatment of the disease, which was once considered to have a grave prognosis.

In conclusion, the present study analyzed patients with breast cancer, who had been diagnosed over the last 21 years at the Oncology Department, Faculty Hospital Trenčín. In line with the published literature, the majority of patients presented with invasive ductal carcinoma with positive hormone receptors. HER 2 positivity was rare. In order to improve knowledge regarding the treatment of patients with this rare cancer, the cases reviewed in the present study should be entered into a prospectively maintained international database. In addition, clinical trials designed for this patient population are required. Alternatively, patients with male breast cancer may benefit from being enrolled into selected clinical trials originally designed for women, where extensive networks of clinical research units already successfully collaborate.

\section{References}

1. Anderson WF, Jatoi I, Tse J and Rosenberg PS: Male breast cancer: A population-based comparison with female breast cancer. J Clin Oncol 28: 232-239, 2010.

2. Mabuchi K, Bross DS and Kessler II: Risk factors for male breast cancer. J Natl Cancer Inst 74: 371-375, 1985.

3. Ewertz M, Holmberg L, Tretli S, Pedersen BV and Kristensen A: Risk factors for male breast cancer-a case-control study from Scandinavia. Acta Oncol 40: 467-471, 2001.

4. NCCN: Genetic/Familial High-RiskAssessment:Breastand Ovarian Cancer, 2015. http://www.nccn.org/professionals/physician_ gls/pdf/genetics_screening.pdf. Accessed January 1, 2016.

5. Müller AC, Gani C, Rehm HM, Eckert F, Bamberg M, Hehr T and Weinmann M: Are there biologic differences between male and female breast cancer explain-ing inferior outcome of men despite equal stage and treatment?! Strahlenther Onkol 188: 782-787, 2012.

6. Yu XF, Yang HJ, Yu Y, Zou DH and Miao LL: A prognostic analysis of male breast cancer (MBC) compared with post-menopausal female breast cancer (FBC). PLoS One 10: e0136670, 2015.

7. Cardoso F, Bartlett J and Slaets L: Abstract S6-05: Characterization of male breast cancer: First results of the EORTC10085/TBCRC/BIG/NABCG International Male BC Program. Cancer Res 75: S6-05, 2015.

8. Sobin LH, Gospodarowicz MK and Wittekind C: The TNM Classification of Malignant Tumours. 7th Edition. Wiley-Blackwell, Geneva, 2009.

9. Diba CS: Cancer incidence in the Slovak Republic. National Health Information Center, Bratislava, 2009. http://www.nczisk. sk/Documents/publikacie/analyticke/incidencia_zhubnych_ nadorov_2009.pdf. Accessed January 2, 2016.

10. Shaaban AM, Ball GR, Brannan RA, Cserni G, Di Benedetto A, Dent J, Fulford L, Honarpisheh H, Jordan L, Jones JL, et al: A comparative biomarker study of 514 matched cases of male and female breast cancer reveals gender-specific biological differences. Breast Cancer Res Treat 133: 949-958, 2012.

11. Leone JP, Leone J, Zwenger AO, Iturbe J, Vallejo CT and Leone BA: Prognostic significance of tumor subtypes in male breast cancer: A population-based study. Breast Cancer Res Treat 152: 601-609, 2015.

12. Senkus E, Kyriakides S, Ohno S, Penault-Llorca F, Poortmans P, Rutgers E, Zackrisson S and Cardoso F; ESMO Guidelines Committee: Primary breast cancer: ESMO clinical practice guidelines for diagnosis, treatment and follow-up. Ann Oncol 26 (Suppl 5): v8-v30, 2015. 
13. Bradley KL, Tyldesley S, Speers CH, Woods R and Villa D: Contemporary systemic therapy for male breast cancer. Clin Breast Cancer 14: 31-39, 2014.

14. Partridge AH, Rumble RB, Carey LA, Come SE, Davidson NE, Di Leo A, Gralow J, Hortobagyi GN, Moy B, Yee D, et al: Chemotherapy and targeted therapy for women with human epidermal growth factor receptor 2-negative (or unknown) advanced breast cancer: American Society of Clinical Oncology Clinical Practice Guideline. J Clin Oncol 32: 3307-3329, 2014.

15. Sasano $\mathrm{H}$ and Harada N: Intratumoral aromatase in human breast, endometrial and ovarian malignancies. Endocr Rev 19: 593-607, 1998.

16. de Ronde W and de Jong FH: Aromatase inhibitors in men: Effects and therapeutic options. Reprod Biol Endocrinol 9: 93, 2011.

17. Nordman IC and Dalley DN: Breast cancer in men: Should aromatase inhibitors become first-line hormonal treatment? Breast J 14: 562-569, 2008.

18. Doyen J, Italiano A, Largillier R, Ferrero JM, Fontana X and Thyss A: Aromatase inhibition in male breast cancer patients: Biological and clinical implications. Ann Oncol 21: 1243-1245, 2010.

19. Bighin C, Lunardi G, Del Mastro L, Marroni P, Taveggia P, Levaggi A, Giraudi S and Pronzato P: Estrone sulphate, FSH and testosterone levels in two male breast cancer patients treated with aromatase inhibitors. Oncologist 15: 1270-1272, 2010.
20. Zagouri F, Sergentanis TN, Azim HA Jr, Chrysikos D, Dimopoulos MA and Psaltopoulou T: Aromatase inhibitors in male breast cancer: A pooled analysis. Breast Cancer Res Treat 151: 141-147, 2015.

21. Di Lauro L, Pizzuti L, Barba M, Sergi D, Sperduti I, Mottolese M, Amoreo CA, Belli F, Vici P, Speirs V, et al: Role of gonadotropin-releasing hormone analogues in metastatic male breast cancer: Results from a pooled analysis. J Hematol Oncol 8: 53, 2015.

22. Clinical trials: S0511, goserelin and anastrozole in treating men with recurrent or metastatic breast cancer. https://clinicaltrials. gov/ct2/show/NCT00217659. Accessed on January 25, 2016.

23. Korde LA, Zujewski JA, Kamin L, Giordano S, Domchek S, Anderson WF, Bartlett JM, Gelmon K, Nahleh Z, Bergh J, et al: Multidisciplinary meeting on male breast cancer: Summary and research recommendations. J Clin Oncol 28: 2114-2122, 2010.

24. de Ieso PB, Potter AE, Le H, Luke C and Gowda RV: Male breast cancer: A 30-year experience in south Australia. Asia Pac J Clin Oncol 8: 187-193, 2012

25. Iorfida M, Bagnardi V, Rotmensz N, Munzone E, Bonanni B, Viale G, Pruneri G, Mazza M, Cardillo A, Veronesi P, et al: Outcome of male breast cancer: A matched single-institution series. Clin Breast Cancer 14: 371-377, 2014.

26. Masci G, Caruso M, Caruso F, Salvini P, Carnaghi C, Giordano L Miserocchi V, Losurdo A,Zuradelli M, Torrisi R, et al: Clinicopathological and immunohistochemical characteristics in male breast cancer: A retrospective case series. Oncologist 20: 586-592, 2015. 